\title{
A Cognitive Approach to Grammatical Mechanism in English Euphemism
}

\author{
Yu Li \\ School of Foreign Languages, Shanxi Normal University, Linfen 041000, China
}

\begin{abstract}
Euphemism is a common linguistic phenomenon. A short introduction to euphemism in English and Chinese will benefit our future intercultural communication. Three grammatical approaches in English euphemism were analyzed in light of cognitive grammar: negation, postmodification and aspect. Thanks to negation, profile and base are changed, resulting in the prominence of "NOT" and the shift of cognitive reference point. Postmodification makes the head noun the trajector in a relational profile, hence reducing the prominence of the modifier. Aspects help to turn a bounded scene into an unbounded one and hence change the cognitive focus. These will be benefit for human's communication.
\end{abstract}

\section{Index Terms - cognitive grammar, euphemistic mechanism, grammatical approach}

The hallmark of cognitive linguistics is the relationship between language and cognition, how humans conceptualize the world around them, how they create and represent meaning and how this is reflected in language. Euphemism is a constantly evolutive linguistic phenomenon in English, which is closely related to culture backgrounds, religious thoughts, life styles, etc. Lots of scholars had studied euphemism from various ways. Such as linguistic, social-linguistic, rhetoric view, pragmatics and so on. When existed euphemism gradually changed into Dysphemism as the increase of usage frequency, new euphemism turned up. Which grammatical mechanism operated in the complicated system of English euphemism? What the role grammatical approaches played?

\section{CUuRrent Research}

It started from Chen Wangdao in domestic research. Hereafter, Chen Yuan, Wu Tieping, Shu Dingfang, Peng Wenzhao and Liu Chunbao analyzed and discussed about it. Early research mainly studied from the point of view of rhetoric, social linguistics, pragmatics and lexicology. Among them, Liu Chunbao (2001) pointed out that grammatical approach was one of constructive methods, but it lacked of further explanation.

As the cognitive linguistics raised, several domestic scholars attempted to interpret grammatical mechanism in Euphemism by using cognitive linguistic theory which was concentrated in cognitive semantics and cognitive pragmatics. The analysis of Shao Junhang and Fan weiwei (2004) was based on predominance and hidden concept, what they thought was that grammatical mechanism depended on transferring listener's focus or dispersing listener's attention, even momentarily interrupting the understanding process of listener. Applied with corpus data, Liang Chunyan (2003) thought that about 59.8\% of euphemism meanings could be analyzed by synthetic space theory. Wang Yongzhong (2003) set about from the category theory in which euphemism put taboo as the collocation of archetypal meanings whose realization has been evolved from archetypal meanings to edged meanings, and has been the typical degree's weakening of family resemblance. Shen Liwen, Zhou Fujuan and Tang Dingjun (2008) respectively investigated the function of conceptual metaphor and conceptual metonymy in euphemism mechanism. Based on space mapping theory and conceptual integration theory. Shen Liwen (2006) analyzed the cognitive differences of euphemism in discourse between English and Chinese. Zhang Ruolan (2009) researched the transfer of conceptual characteristics and the acceptability of euphemism meanings.

At the same time, it was much less for using cognitive linguistics to interpret euphemism in abroad researches. Migual (2009) put forward that euphemism wasn't linguistic phenomenon constricting in vocabulary level, but a cognitive progress using various grammatical methods to conceptualize taboo facts.

All suggests that current research has certain limitations: 1. Analysis was concentrated on vocabulary level; 2 . Perspective was confined in macro discussion by using metaphor, metonymy and conceptual integration; 3.The quantity of exemplification was limit and repetitive. Euphemism approach was one kind of cognitive mechanism, which was based on experiencing philosophy, investigated language from cognitive feature, focused on finding motivation of grammatical structure. It was limit that human interpreted English euphemism approach from the perspective of cognitive grammar. Thus, under the framework of cognitive grammar, this text discussed the grammatical approach in euphemism approach based on realistic English corpus.

\section{INTERPERSONAL FUNCTION OF EUPHEMISM}

The function of language is realized through communication so that language is considered as the most important communicative tool. Research on the function of language can help us understand how language plays its role in the macro 
human society. Languages play different parts in different social communications and bear their unique features on different levels of languages. The function of language in the language system is realized by people's choosing different meaning potentials under different social and cultural background.

American scholar Rawson (1983) states: "Euphemisms are embedded so deeply in our language that few of us, even those who pride themselves on being plainspoken, ever get through a day without using them" (p1). Generally speaking, words that are subtle, indirect or nice are euphemisms. Allan and Burridge (1996) divide euphemism into euphemism, dysphemism and orthophemism. Rawson (1983) suggested that euphemisms can be divided into positive euphemisms and negative euphemisms. Euphemism selected by people in the process of communication is affected by a variety of factors such as context, cultural differences of the parties involved in the communication, social cognition and so on. While at the same time, it dynamically reflects the interpersonal function of language. In the course of human's stepping into civilization, euphemism is virtually the product of language adapting to society and interacting to society. Euphemism bears rich social and cultural connotation and touches upon every aspect of social life such as religion, politics, literature and linguistics. Euphemism employs the fuzziness of language to reconcile the contradiction in social relationship and interpersonal relationship. With the continuous development of social life and social relationship, the interpersonal function of euphemism will undergo alteration and metabolism to adjust to the development and change. Therefore, we should choose different euphemisms to better achieve the objective of communication and bring the interpersonal function of euphemisms into full play according to different context.

When it comes to euphemism, how many ways can we use to achieve the effect of euphemism? Are grammatical approaches the methods realizing the effect of euphemism? As a necessary part of English, grammar plays an important role during studying. But which grammatical mechanism operated in the complicated system of English euphemism?

\section{Cognitive Construal And Negation ApProach}

The cognitive approach is an approach to foreign language teaching which is based on the belief that language learning is a process which involves active mental process and not simply the forming of habits. As the Audiolingual Method was on the decline in the 1960s, the cognitive approach developed as an alternative, in response to the criticisms leveled against audiolingualism. In the meantime the structural linguistics gave way to the generative linguistics that turned the attention from mechanistic conditioning to meaningful leaning. When it comes to cognitive approach, most people take memory for granted, and the fact that people remember more often than they forget tends to lead us to overlook the underlying complexity of memory as a cognitive process. Others think that the basic question about cognitive processes is the relationship between thinking and language. But now we will analyze grammatical mechanism from the perspective of cognitive construal.

Langacker's Construal shows one kind of cognitive capacities that people choose different scopes, make different focus prominent, use various methods to observe situation and explain contents from different perspectives for achieving the aim of thinking and expressing. Construal could be depicted from degree of specificity, scope, background, perspective and salience prominence. In these aspects, nothing is better than scope, perspective and salience prominence which are familiar with euphemism ( Langacker, 2008).

An expression's 'scope' comprises the full array of conceptual content that it specifically evokes and relies upon for its characterization. The term 'lid,' for instance, evokes the schematic conception of a container, as well as the notion of one entity covering another. A conception of any type or any degree of complexity is capable of being invoked as part of an expression's meaning. Perspective means angles for describing affairs. It can directly influence understanding and expressing because different perspectives produce different reference points. The choosing of perspective is closely related to background and salience prominence. The selection of the clause subject is determined by the different degrees of prominence carried by the elements involved in a situation. This prominence is just reflected in the selection of the subject as opposed to the object and the adverbials of a clause, but there are also many other applications of what may be called the prominence view of linguistic structures. The prominence view provides the explanation of how the information in a clause is selected and arranged. Salience prominence can be categorized into two parts. One is profile-base; the other is trajector-landmark. The former describes the meaning and the later depicts relational profile. The negative approach of euphemism is mainly connected with the former. Base is one relative cognitive domain with which expressions covered. One word's base just is the scope involved in related cognitive domain and the foundation of meanings. Profile means one part which was the biggest highlighted in language body, the focus in base, and the semantic structure which words identified. Base is the starting point of the profile description; profile is focus of specific contents, identifying base's directive property. The meaning of word depends on the combination of base and profile.

In English negation is not simple addition of negative word. It involves many factors. Some negation is constructed by negative prefixes. The most frequently used negative affixes are un-, non-, dis-, in-, mal-, mis-, anti-, counter-, -less, etc. Non- can normally be regarded as corresponding to clause negation: non-smoker means one who does not smoke. It frequently contrasts with un- in expressing binary contrast, rather than the opposite end of a scale.

There are quite a number of euphemisms in English; they are showed as vocabulary substitution in the form. In reality they change views with the help of negative approach, transform the base through changing profile of prominence, replace reference point, finally attain euphemism of semantics. Take "disease" as example, in the beginning, it is a euphemism when Chaucer first used. As 1(a) shown in the figure, the profile of disease is "not" (Dis), 
base is "ease". Through the negative meaning of "dis", Chaucer made "ease" as cognitive reference point. Accordingly, the combination of base and profile turned into "not ease" which is relative to "ease". By contrast, in the specific name of "headache", profile is "ache", base is "head". (See chart b) The combination of base and profile highlight having an explicit head pain. In other words, "dis" successfully replaces the cognitive reference point, transforms base into acceptant commendatory concept and makes general concept "not" prominent, which results in euphemism and abstraction.

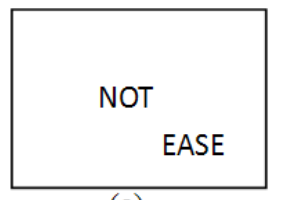

(a)

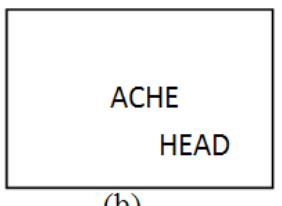

(b)

Figure1 the profile and base of negative approach

It is common that using negative approach to reach the effect of euphemism. The euphemism "illness" can be expressed as disease, disorder, infirmity, indisposition, unwell and so on. In addition, similar expressions such as disabled (crippled), imperfect (deaf), disadvantaged (poor), underprivileged (poor) are too numerous to enumerate. All these expressions use negative approach to change reference point and highlight general concept of "not" or "not enough" (under), which reach an effect of euphemism. Thus, it can be seen that negative approach could effectively guide people's cognitive construal. So we can achieve the communicative goal of euphemism.

\section{Quantitative COGNitive ApProach AND Postmodification ApProACH}

Premodification is an intensified order in English, so originally postmodification have showed the tendency of preposition during development of language. For example, a heart-disease specialist (a specialist in heart disease), Domestic-Affair Department (department of Domestic-Affair), a what's-to-be-done-next look was still written on his face (a look that shows What's to be done was still written on his face). However, euphemism goes in the opposite direction where they are expressed as postmodification from premodification. For example, it is "people with vision impairment" that substitute "blind people".

In fact, America once advocated "people first" activity to change title for people with disabilities (Helena, 2011), which demanded people to use head-word "people+postmodification" to referring people with disabilities. For example, using "person with (who has/having) a disability" to replace "disabled person", "person with mental illness" to substitute "mentally ill person". This kind of action was comprehensively popularized in the field of psychology and pedagogy. Helena who was based on the corpus of Google and The Houston, specially conducted a study about the actual usage of "people first". It has shown that authority advocated the way of postmodification, but people largely used non-postmodification expressions (non-PC). The postmodification to non-postmodification was 3 to 7 . Apart from striving to the simplicity of news and diversity of expressions, Helena found the rules for using occasions about premodification and postmodification. The premodification expressions generally referred to non-popular members such as criminal, fictional character. The postmodification expressions referred to children and well-behaved adults. People selectively adopted postmodification when in need of euphemism. It concluded that postmodification would reach the effect of euphemism. Clearly, changing of position from premodification to postmodification concealed deeper cognitive differences what postmodification used to achieve euphemism. Take "disabled person-person with a disability" for instance, as the only modifier of person, the meaning of "disabled" restricted "person", making cognition focus on the only character "disabled". It made that simplex and partial distinction was amplified as overall quantitative distinction. Finally the semantic of disabled was naturally highlighted. As for "person with a disability", it presented the relational profile above "with" to us through postmodification. The participants "person" and "disability" were asymmetric in relational profile. In the most prominent location, the trajector "person" who was a starting point of relational profile was initial focus and first participant. While "disability" that was the subordinate location and provided orientation for trajector with reference point was landmark. Thus, the cognition of "person" was intensified while "disability" relatively was attenuated. Through prominent relational profile, postmodification presented a fact that the coexistence of "person" and "disability", which avoided making qualitative study for its entirety by means of simple feature. The same euphemism reflected in "person who has/ having a disability".

As for cognitive process, postmodification is more complex in the aspect of constitution. Even if euphemism approach based on trajector-landmark isn't taken into account, the cognitive difficulty of euphemism carrying with postmodification is larger than the corresponding premodification from point of vocabulary. To increase cognitive difficulty is just the effective way for achieving euphemism. Moreover, benefited by postmodification, some euphemisms use more complex or obscure expression in postmodification to increase cognitive difficulty. Such as people who are mobility impaired (cripples), person with symptoms of mental illness (crazy, paranoid), child with syndrome (mongoloid) and so on.

\section{BOUND THEORY AND GRAMMATICAL APPROACH OF ASPECT}


The term aspect refers to a grammatical category which reflects the way in which the verb action is required or experienced with respect to time. In its broadest possible interpretation, the perfective indicates anterior time, i.e. time preceding whatever time orientation is signaled by tense or by other elements of the sentence or its context. The present perfective signifies past time with current relevance while the past perfective usually has the meanings of past-in-the-past, and can be regarded as an anterior version either of the present perfective or of the simple past. On the other hand, the progressive presents the situation as being in progress. This implies that the situation is concerned of as having a more or less dynamic character, as opposed to being wholly static. The progressive form in English is closely related to the idea of incompletion. It distinguishes acts and events, which are complete, from activities and processes, which are not.

Aspect in English is an important grammatical approach that helps verb realize the switch from bounded scene and unbounded scene (Declerck, 2011). According to Modern Linguistics Dictionary (Crystal, 2000), aspect is a category for verb grammatical description, which mainly means length of time and the types marked by verb. Obviously, the length of time of marked verb is the main function of aspect. Progressive aspect and Perfect aspect are two types in English verb. As far as aspect, progressive aspect shows the continuity, temporary, accomplishment of action, so it is unbounded. Perfect aspect presents the accomplishment and endness of action, so it is bounded. Another explanation shows that aspect actually interacts with some already existing semantic features making the interpretation complete. One of such approaches is that perfective aspect typically presents the situation in its entirety, as a whole and imperfective as a structure or, in other words, that aspect deals with the inclusion or exclusion of the endpoints of a given situation. Step by step, this could bring us to the notion of boundedness which means that one can start from an assumption that perfective aspect implies a bounded, while the imperfect aspect unbounded situation.

In order to know bounded and unbounded scene, consider the examples below:

a. Helen was filling the bottle with water for ten seconds.

b. Helen filled the bottle with water in ten seconds.

The progressive aspect and durational adverbials implies that (a) is telic unbounded while (b) is telic bounded.

"Bounded and unbounded" is a pair of important concepts in cognitive grammar. In cognitive grammar, "bounded and unbounded" is part of general cognitive mechanisms. People acknowledge what the bounded is from their own body, then know what unbounded is with the help of the opposition between bounded and unbounded (Shen, 1995). Reflecting on language, they are expressed by countability or uncountabiliy of noun, continuity or discontinuity of verb, property or character of adjective. In terms of action, the occurrence and continuity of it need take up time, which results in the differentiations between bounded and unbounded. Bounded action has starting point and final point on time axis, and unbounded doesn't. The specific presentations of them, what Langacker (2008) points out, are that the inside of bounded action is heterogeneous and replicable, and the inside of unbounded action is homogeneous and unrepeatable. Shown in English, typical continuous verbs have like, need, know and so on, which refer to unbounded action, while typical discontinuous verbs have jump, go, arrive and so on, which refer to bounded action.

As same as action, based on whether there is an end, scene can be categorized into two types: bounded and unbounded.

For example, 1. Betty made a drawing on the floor.

2. (1) The athlete ran 10 kilometers.

(2) a. The athlete ran.

b. John was in the kitchen.

c. Betty knew about our affair.

From the above examples, sentence1 presents a kind of bounded scene. Sentences2 (2) presents a kind of unbounded scene. It's worth noting that sentence 2(1) is bounded and sentence 2(2.a) is unbounded, although they use the common verb "run". Thus, the same verb in a sentence, under the effect of other grammatical approaches, could change in boundedness which they referred to.

Something is to be pointed out, "bounded and unbounded" reflect people's knowledge rather than objective reality. Through different "aspect", English euphemism uses this factor to guide people's cognition achieving the effect of euphemism in the end. The typical example is "developing". Developing is same as underdeveloped and undeveloped, describing the lower standards of countries and regions. Compared to the directive "poor", undeveloped and underdeveloped have already achieved the transfer of cognitive focus from poor to "developed" with the help of negative approach, which results in certain degree of euphemism (Wang, 2006). Not only undeveloped, but also underdeveloped originated from verb "develop". Both of them are in the form of the complete body, presenting a bounded scene. They make incomplete "developed" as reference, highlight the character of end. As figure 2 is showing, under the guidance of two words, the observer cuts-over from "now", observes a finished action at present (developed) and correspondingly stoical results (undeveloped or underdeveloped). Developing is benefited from progressive aspect highlighting unbounded action in progress. In view of homogeneity of unbounded action, the action developing referred to is homogeneous at any time on axis. That is to say, seeing from current perspective, the action of "develop" is ongoing at special time of "now". When jumping out of time node "now", the action of "develop" is also underway from past to present, from present to future. Therefore, from perfect aspect to progressive aspect, developing makes a good guidance for people's cognitive process, achieves the transfer from bounded scene to unbounded scene and 
highlights the sustainable development process and future prospects of developing/ undeveloped, underdeveloped countries and regions, which results in the effect of euphemism. In fact, all these three words which collocate with country, nation and area refer to the same meaning. But based on the statistical results of COCA corpus in America, the usage frequency of developing is often higher than others. It owes to the effect of euphemism which grammatical approach "aspect" has.

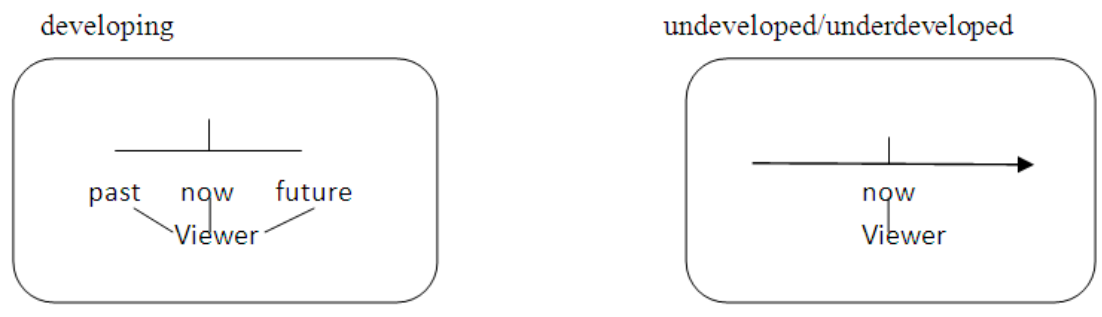

Figure2. the bounded and unbounded scene of Aspect

\section{CONCLUSION}

As the rapid development of science and technology, our world becomes a global village. People from all around the world have a stronger tie than ever before. Intercultural communication turns out to be a hot topic. Every day, every time, we are having with people in various backgrounds. The application of euphemism has become an indispensable part of social life. An appropriate grammatical approach is selected in communication. The process of analysis of grammar is communication is actually a mental cognitive process of communication affected by cognitive contexts. Under the perspective of cognitive grammar, euphemism guides people's cognition through the features of language negation, postmodification and aspect. It effectively achieves the transfer of cognitive focus and finally realizes the effect of euphemism through different operation mechanisms. Thanks to negation, profile and base are changed, resulting in the prominence of "not" and the shift of cognitive reference point. Postmodification makes the head noun the trajectory in a relational profile, hence reducing the prominence of the modifier and avoiding overall qualitative research with a single partial feature. Aspects help to turn a bounded scene into an unbounded one and hence change the cognitive focus, finally realize the effect of euphemism.

The language we use is a process which involves active mental processes and not simply the forming of habits. Cognitive approach gives importance to the learner's active part in the process of using and learning of grammatical rules. A full view of euphemism is necessary in our daily life. When we learn to express us, a full knowledge of euphemism can avoid embarrassment in communication with multi-background people. Therefore, we should choose the appropriate euphemism according to the concrete context so as to make the communication smoother and the interpersonal relationship more harmonious. With the continuous development and changes of the social life and social relationship, the interpersonal function of euphemism will undergo alteration and metabolism to adjust to the development and change. It manifests that the interpersonal function of euphemism bearing strong vitality and will proceed to play immeasurable role in social life.

\section{REFERENCES}

[1] Allan, K \& Burridge, K. (1993). Forbidden words: Taboo and the censoring of language. NewYork: Cambridge University Press.

[2] Crystal, D. (2000). Modern Linguistics Dictionary. Beijing: Commercial Press.

[3] Declerck, R. (1991). Tense in English. London and New York: Routledge.

[4] Helena Halmari. (2011). Political correctness, euphemism, and language change: The case of 'people first'. Journal of Pragmatics, $43,828-840$.

[5] Kan Liwen.(2006). Cognitive motivation of conceptual metaphor and euphemism metaphor in meaning construction. Foreign Language and Foreign Language Teaching, 8, 17-20.

[6] Liang Chunyan.(2003). The explanatory power of blended space theory for euphemisms. Journal of Jinan University, 2, 53-61.

[7] Liu Chunbao.(2001). English euphemism dictionary. Beijing: Commercial Press.

[8] Miguel Casas Gomez. (2009). Towards a new approach to the linguistic definition of euphemism. Language Sciences, 31, 725-739.

[9] Rawson, H.(1983). A dictionary of euphemisms and other doubletalk. London: Macdonald \& Co (Publishers) Ltd. Retrieved Sept. 24, 2007 from http://www.doe.state.in.us/lmmp.

[10] Ronald W. Langacker. (2008). Cognitive Grammar: A basic introduction. New York: Oxford University Press.

[11] Shao Junhang, Fan Weiwei.(2004). Cognitive linguistics interpretation of euphemism mechanism. Foreign Language Research, 4 , 20-25.

[12] Shen Jiaxuan. (1995). Bounded and unbounded. Chinese Literatures, 5, 367-389.

[13] Wang Yongzhong. (2003). Cognitive motivation of euphemismin the perspective of category. Foreign Languages and Literatures, 2 , 3-5.

[14] Wang Yan. (2006). An introduction to cognitive grammar. Shanghai: Shanghai Foreign Language Education Press.

[15] Zhang Ruolan. (2008). Interpretation of euphemism based on conceptual feature transfer and accessibility constraint. Foreign Language Teaching, 4, 50-53.

[16] Zhou Fujuan, Tang Dingjun. (2008). Conceptual metonymy as cognitive motivation in English euphemisms. Foreign Language 
Research, 5, 34-36.

Yu Li was born in HongTong, Shanxi in 1995. She received her bachelor's degree in English from LvLiang University, Shanxi in 2016. She is currently a postgraduate studying for her master's degree and majoring in Foreign Linguistics and Applied Linguistics in Shanxi Normal University. Her research interests included cognitive linguistics and English learning strategies. 